\title{
Impacto ambiental en los recursos naturales de una zona afectada por la actividad minera en Bolivia: evaluación geoquímica de suelos y sedimentos
}

\author{
José A. Acosta, Angel Faz, Mª́ngeles Muñoz, María Gabarrón, Silvia Martínez-Martínez
}

Grupo de investigación "Gestión, Aprovechamiento y Recuperación de Suelos y Aguas (GARSA)", Universidad Politécnica de Cartagena. Paseo Alfonso XIII, 48, 30203. Cartagena, España.

ja.acosta@upct.es

\begin{abstract}
RESUMEN
Los métodos actualmente utilizados en Bolivia para extraer oro, a través del proceso de amalgamación con mercurio, ocasionan tanto contaminación ambiental como riesgo para la salud. El objetivo de este trabajo fue determinar las concentraciones de $\mathrm{Hg}, \mathrm{As}, \mathrm{Cu}, \mathrm{Cd}, \mathrm{Pb}$ y $\mathrm{Zn}$ en suelos y sedimentos en tres distritos mineros. Los resultados mostraron que en el Distrito Minero Sunchullí-Viscachani las concentraciones de As y $\mathrm{Hg}$ fueron debidas a una activa deposición atmosférica de $\mathrm{As} / \mathrm{Hg}$, cuyas concentraciones excedieron los niveles de referencia. De igual modo, las concentraciones de $\mathrm{Cd}$ y $\mathrm{Zn}$ superaron los niveles de referencia en los distritos mineros de Katantica y Sural. Por su parte, los sedimentos de las lagunas Sunchullí y Viscachani estaban contaminados por $\mathrm{Hg}$, As, Cd, $\mathrm{Zn}$ y Cu, mientras que la laguna Katantica mostró altas concentraciones de $\mathrm{Zn}, \mathrm{Cd}$ y $\mathrm{Hg}$, siendo las concentraciones de As, $\mathrm{Zn}$ y Cu elevadas en la laguna Sural. En relación a los ríos estudiados, el río Sunchullí presentó altas concentraciones de $\mathrm{Cu}, \mathrm{Pb}, \mathrm{As}$ y $\mathrm{Hg}$; el río Pelechuco de $\mathrm{Hg}$ y $\mathrm{Cu}$, y el río Rayo Rojo de As y Hg, en todos los casos procedentes de los vertidos directos de la actividad minera. Por lo tanto, tanto las concentraciones encontradas en los ríos como en las lagunas podrían estar causando un cierto impacto tanto en los ecosistemas acuáticos como en la salud de la población que se abastece de las aguas de estos ríos. Por lo tanto, se recomienda estudiar en detalle la disponibilidad y especiación de metales y metaloides en los suelos y sedimentos para comprender mejor el ciclo de estos elementos y su transferencia a la cadena alimentaria.
\end{abstract}

Palabras clave: amalgamación, mercurio, metales, metaloides, minería de oro.

\section{Environmental impact on the natural resources of an area affected by mining activity in Bolivia: a geochemical evaluation of soils and sediments}

\begin{abstract}
The current methods used in Bolivia to extract gold using the amalgamation process with mercury result in environmental contamination and a risk to human health. The objective of this study was to determine $\mathrm{Hg}$, $A s, \mathrm{Cu}, \mathrm{Cd}, \mathrm{Pb}$ and $\mathrm{Zn}$ concentrations in soils and sediments from three mining districts. Results showed As and $\mathrm{Hg}$ concentrations were higher in the topsoil than in the sub-surface soils in the Sunchulli-Viscachani area, indicating active $\mathrm{As} / \mathrm{Hg}$ atmospheric deposition, where concentrations exceeded the threshold levels. In addition, concentrations of $\mathrm{Cd}$ and $\mathrm{Zn}$ largely exceeded the threshold levels in the Katantica and Sural mining districts. In addition, the Sunchullí and Viscachani lagoon sediments were contaminated by $\mathrm{Hg}, \mathrm{As}, \mathrm{Cd}, \mathrm{Zn}$ and $\mathrm{Cu}$, whilst Katantica lagoon showed high concentrations of $\mathrm{Zn}, \mathrm{Cd}$ and $\mathrm{Hg}$, and high levels of $\mathrm{As}, \mathrm{Zn}$ and $\mathrm{Cu}$ were reported in the Sural lagoon. Our results showed that the $\mathrm{Cu}, \mathrm{Pb}, \mathrm{As}$ and $\mathrm{Hg}$ concentrations in sediments in the Sunchulli river; $\mathrm{As}, \mathrm{Hg}$ and $\mathrm{Cu}$ in the Pelechuco river, and $\mathrm{As}$ and $\mathrm{Hg}$ in the Rayo Rojo river all come from direct mine discharges into the rivers. These high concentrations of metals and metalloids must cause an
\end{abstract}


impact on the aquatic ecosystems and human health. Therefore, there is a need to study the availability and speciation of metals and metalloids in soils and sediments to better understand the cycling of these elements and their transfer to the food chain.

Keywords: metalloids; metals; amalgamation; mercury; gold mining.

\section{ABRIDGED ENGLISH VERSION}

\section{Introduction and Methodology}

The mining industry, particularly the extraction of $A u$ and $\mathrm{Zn}$, has always been one of the pillars of the national economy of Bolivia (Loayza and Franco 2000). Currently gold is being extracted using an $\mathrm{Hg}$ amalgamation technique that represents a highly economical and practical technique, which has been used since 1556 (Enríquez 2001). Hg amalgamation is based on the formation of gold-Hg alloy, which is subsequently heated to volatilise $\mathrm{Hg}$ to obtain pure gold. However, depending on the mining company, $\mathrm{Hg}$ is not always recovered and $5-45 \%$ of the total $\mathrm{Hg}$ used can be released into soil, water and the atmosphere (Malm et al., 1990; Maurice - Bourgoin et al., 1999). In addition, arsenopyrite (FeAsS) minerals, which commonly occur with the Au ore deposits, are likely sources of high As in soil. Therefore, pollution of soil, water, and air surrounding mine areas poses threats to ecosystems and human health (Faz et al., 2003). Mercury is among the most highly toxic trace metal in the food chain, and many national and international agencies and organizations are interested in developing methods for its possible emission control (Horvat et al., 2003). The elemental $\mathrm{Hg}(\mathrm{HgO})$ can transport from its source to the final receptor following a multi-step frequency, which includes emission to the atmosphere, transportation and deposition. Arsenic in Bolivia is present mostly in the west Andean region, specifically in the zone known as the "As-Ag-Pb-Zn-Cu-Au belt" (Litter et al., 2010), where high levels of natural enrichment by arsenic are found (MDSMA, 1996). Inorganic As is toxic and can cause cancer in skin, lungs, urinary tracts, cardiovascular disease, neurotoxicity and diabetes (WHO, 2014). Therefore, the aims of this study have been to characterize the contamination of $\mathrm{Hg}$, $A s, \mathrm{Cu}, \mathrm{Cd}, \mathrm{Pb}$ and $\mathrm{Zn}$ in soils and sediments and to assess the risk to human health and the environment in different mining districts in the highlands of Bolivia.

Three different gold mining districts affected by metal and metalloid pollution were selected: Sunchullí-Viscachani, Katantica, and Sural (Fig. 1). Four soil profiles were collected in the Sunchulli-Viscachani area, where profiles I and II were located $6 \mathrm{~km}$ north-west of the Sunchulli Lake and $18 \mathrm{~km}$ from the main mining operations, on the north side of the mountain range (Fig. 1); profiles III and IV were located less than $500 \mathrm{~m}$ from the main mining operation. In addition, in order to evaluate the accumulation of metals and metalloids on the bed of the water bodies, seven sediment samples were taken: three from the Sunchulli lagoon, two from the
Viscachani lagoon, and four from the Sunchulli River. The Katantica mining district is located in the northern part of the Apolobamba gold mine area. In this area, one soil profile (Profile l) located $1 \mathrm{~km}$ from the mining operation was sampled, in addition, two sediment samples from the Katantika lagoon, two from the Pelechuco river, one from Rayo Rojo river and one from the Turcos river were collected. Finally, from the Sural mining district, which is located in the north-western part of Apolobamba, two profiles were sampled, both profiles were taken $5 \mathrm{~km}$ south-east from the Sural lagoon and $1 \mathrm{~km}$ east from Sural river, in addition, two sediments samples from the Sural lagoon were collected. Soils and sediments were characterized for $\mathrm{pH}$, organic carbon, total nitrogen, electrical conductivity, particle size distribution, and cation exchange capacity. An advanced $\mathrm{Hg}$ analyser was used, and total $\mathrm{Pb}, \mathrm{Cu}$, $A s, \mathrm{Zn}$, and Cd were analyzed using an atomic absorption spectrophotometer.

\section{Results and Discussion}

Soils in the three mining districts were from strongly acid to neutral (Soil Survey Division Staff 2006), which promotes metal solubilisation, and increases metal mobility (Kabata-Pendias and Pendias 2010). Soils were non-saline in the three mining districts (USDA 2003). TOC, TN and CEC showed the highest values in the topsoil of the profiles from the three mining districts, probably due to the high vegetation cover found of these areas (Table 1). However, the decreased TOC and TN in areas near the mining operation sites could cause the extinction of the plant cover affecting the soil stability and promoting soil erosion processes. The $\mathrm{pH}$ values in the lagoons were neutral in Sunchulli, moderately acid in Viscachani, slightly acid in Sural and from strongly acid to neutral in Katantica (Soil Survey Staff, 1993). All the sediments were non-saline (Soil Survey Division Staff, 1993). TOC and TN concentrations of the sediments from Viscachani were the highest, whilst in the Sunchilli lagoon they were the lowest, probably due to the contribution of the vegetation (Table 2). The $\mathrm{pH}$ of the river sediments ranged from very strong acid in the Sunchulli river to slightly alkaline in the Pelechuco river) (Soil Survey Division Staff, 1993). The sediments of all the rivers were not saline (Soil Survey Division Staff, 1993). CEC, TOC and TN concentrations of the river sediments were lower than those reported from the lagoon sediments (Table 3).

The results from the Sunchulli-Viscachani profiles (Table 4) suggest that atmospheric deposition of $\mathrm{Hg}$ 
and As may occur in this area, however concentrations in profile I represent the natural accumulation of $\mathrm{Hg}$ and As in the topsoil. Oppositely, profile II, III and IV showed much higher concentration in the topsoil than subsoil, indicating active atmospheric deposition of $\mathrm{Hg}$ and As. Mercury can be transported in small particles and cause problems in the breathing system (Peñaloza and Reinhardt, 2000). These particles could reach agricultural soils and pastures near to the mine areas by atmospheric transport. Concentrations of $\mathrm{Pb}, \mathrm{Cu}$, and $\mathrm{Zn}$ were higher in profile II than in profile I, probably this difference is due to the natural occurrence of $\mathrm{Pb}$. There is a tendency of $\mathrm{Pb}$ to concentrate in the felsic series of magmatic rocks and argillaceous sediments, in addition, the concentration of $\mathrm{Pb}$ in $\mathrm{C} / \mathrm{R}$ horizon in profile Il is similar to the $\mathrm{Pb}$ concentration in the superficial horizon and supports this hypothesis. In addition, total $\mathrm{Pb}, \mathrm{Cu}$ and $\mathrm{Zn}$ concentrations are similar in profiles III and IV and show an increase with depth for $\mathrm{Pb}$ and $\mathrm{Cu}$, indicating a geogenic origin of these metals. $\mathrm{Hg}, \mathrm{As}$, $\mathrm{Pb}$ and $\mathrm{Cu}$ concentrations in the Katantica and Sural areas (Table 4) was lower than in the Sunchulli-Viscachani. Profile samples showed values of $\mathrm{Hg}, \mathrm{As}, \mathrm{Pb}$ and $\mathrm{Cu}$ lower than the reference concentrations given by the commonly used international regulation. However, concentrations of $\mathrm{Cd}$ and $\mathrm{Zn}$ were higher than in the Sunchulli-Viscachani area and largely exceeded the thresholds levels, indicating a higher risk for humans and the environment, especially for $\mathrm{Cd}$, though their origin must be geogenic since the highest concentrations were reported in the subsoil.

Most of the sediments from the Sunchulli and Viscachani lagoon were contaminated by $\mathrm{Hg}, \mathrm{As}, \mathrm{Cd}, \mathrm{Zn}$ and $\mathrm{Cu}$ (Table 5). The $\mathrm{Hg}$ and As concentrations found in the sediments probably come from primitive amalgamation processes through direct spill of waste to the water bodies. No enrichment was found for $\mathrm{Pb}$, $\mathrm{Cu}$ and $\mathrm{As}$ in the sediments of the Katantica lagoon. However, sediments located near the mining operations exhibited $\mathrm{Hg}$ enrichment (Table 5). In the same way, high concentrations of $\mathrm{Zn}$ and $\mathrm{Cd}$ were reported in both sediments collected from this lagoon, indicating that the sediments are important sinks for these metals. $\mathrm{Hg}, \mathrm{Pb}$ and $\mathrm{Cd}$ concentrations in the sediments from Sural lagoon were lower than the levels proposed by the used legislation (Table 5). However, $\mathrm{Zn}, \mathrm{Cu}$ and As concentrations exceeded these levels, indicating an enrichment of these elements in the sediments, and a potential risk for the ecosystem of the lagoon.

Our results showed that total $\mathrm{Cu}, \mathrm{Pb}, \mathrm{As}$ and $\mathrm{Hg}$ content in the sediments from the Sunchulli river come from direct discharges (Table 6). These discharges of $\mathrm{Pb}$ and $\mathrm{Hg}$ ( $\mathrm{Cu}$ and $\mathrm{As}$ ) from mine operations may have a significant medium and long-term impact on the environment. We should mention that many settlement areas are located in the downstream zone where water is consumed for domestic or irrigation purposes, and therefore may be subject to significant health risks. In the Pelechuco river, due to the cumulative contribution of the spills along the river, sediments have high concentrations of $\mathrm{Hg}, \mathrm{As}$ and $\mathrm{Cu}$. Finally, higher concentrations of $\mathrm{Pb}, \mathrm{Cu}, \mathrm{As}$ and $\mathrm{Hg}$ in the sediments from Rayo Rojo river were found than in the Turcos river, however only $\mathrm{As}$ and $\mathrm{Hg}$ exceeded the recommended level proposed by the legislation.

\section{Introducción}

La industria minera, particularmente las extracciones de $A u$ y $Z n$, siempre han sido uno de los pilares fundamentales de la economía nacional en Bolivia (Loayza y Franco, 2000). Durante décadas, el Cerro Rico de Potosí constituyó el yacimiento de minerales de plata más importante del mundo. Para aprovechar la plata de Potosí, a partir de 1545 se fueron introduciendo tecnologías punteras para su tiempo; pero no se consideraron ni los efectos para la población originaria ni los impactos sobre el medio ambiente. El "Área Nacional de Manejo Integrado de Apolobamba (ANMINA)" ubicada en la parte noroeste del Departamento de La Paz, Bolivia, es una de las más importantes regiones mineras para la extracción de oro. Actualmente, el oro se extrae utilizando la técnica de amalgamación de $\mathrm{Hg}$, técnica que ha sido usada desde 1556 (Enríquez 2001). La amalgamación de $\mathrm{Hg}$ se basa en la formación de una aleación de Au-Hg que posteriormente es calentada para volatilizar el $\mathrm{Hg}$ y obtener oro puro. Sin embargo, el $\mathrm{Hg}$ no se recupera en su totalidad y entre el $5-45 \%$ del $\mathrm{Hg}$ total utilizado puede ser liberado al medio ambiente, con los consecuentes riesgos para el suelo, agua y atmósfera (Malm et al., 1990; Maurice-Bourgoin et al., 1999). Normalmente, entre 0,5 y $1 \mathrm{~kg}$ de $\mathrm{Hg}$ se volatiliza y pasa a la atmósfera para obtener $1 \mathrm{~g}$ de $\mathrm{Au}$, donde la mayor parte es condensado y precipitado en las proximidades de las zonas de procesado (Wotruba et al., 1998). De igual modo, asociada a los depósitos de oro, se encuentra la arsenopirita (FeAsS), mineral que es fuente de As, el cual también puede llegar a contaminar suelos, agua y sedimentos. Además, la oxidación tanto de minerales que contienen azufre como los residuos de la actividad minera genera un drenaje ácido, el cual favorece la solubilización de elementos potencialmente tóxicos como As, Sb, Zn y Cd. Por lo tanto, la contaminación de los suelos, el agua y el aire que rodean las áreas mineras representa una amenaza para el ecosistema y la salud humana de estas zonas mineras y sus alrededores (Faz et al. 2003).

Es en el siglo $X X$, en la década de los 80 y principios de los 90 cuando se despierta el interés por la problemática ambiental mediante la realización de diversos estudios sobre impactos ambientales causados por la actividad minera. Durante ese periodo, la Secretaría Nacional de Medio Ambiente (SENMA) Ilevó a cabo una evaluación de los sectores minero e industrial en Bolivia, y concluyó que los principales impactos en el medio ambiente de la actividad minera son: el consu- 
mo de recursos no renovables y escasos, principalmente el uso de la tierra y del agua; la transformación del paisaje; la contaminación de aguas superficiales, aguas subterráneas y agua del suelo; la acumulación de residuos sólidos; y la emisiones de polvo que contiene metales pesados que pueden llegar a contaminar el agua y los suelos (Enríquez, 2001). De igual modo, otros estudios encontraron cierta contaminación de $\mathrm{Hg}$ en sedimentos y aguas de ríos (Maurice-Bourgoin et al., 2001), e incluso en peces (Alanoca et al., 2000).

El Hg se encuentra entre los metales traza más tóxicos de la cadena alimentaria, y muchas administraciones y organizaciones nacionales e internacionales están interesadas en desarrollar métodos para el posible control de sus emisiones (Horvat et al., 2003). La transferencia y el transporte de $\mathrm{Hg}$ ha sido estudiado en detalle $\mathrm{y}$ se tiene un amplio conocimiento del mismo (USEPA, 2000). El Hg elemental $\left(\mathrm{Hg}^{0}\right)$ puede llegar desde su origen hasta el receptor final siguiendo una frecuencia de varios pasos, que incluye la emisión a la atmósfera, el transporte y la deposición. Travnikov (2005) estudió la dispersión de $\mathrm{Hg}$ en la atmósfera del hemisferio norte y sugirió que la contribución de las fuentes antropogénicas y naturales a la deposición de $\mathrm{Hg}$ en diferentes continentes varía entre el $30 \%$ y el $70 \%$. En general, el $\mathrm{Hg}$ presenta una fuerte volatilidad en la mayoría de las condiciones atmosféricas (Zang-Ho et al., 2005).

Por su parte, el arsénico en Bolivia está presente principalmente en la región andina occidental, específicamente en la zona conocida como el cinturón "As-Ag-Pb-Zn-Cu-Au" (Litter y Morgada, 2010), donde altos niveles de contaminación natural por arsénico han sido atribuidos a una intensa actividad magmática desde el Mioceno (MDSMA, 1996). Estas elevadas concentraciones de As en el suelo pueden ser movilizados y alcanzar otras áreas llegando a contaminar aguas, sedimentos y biota. De igual modo, el As es tóxico para la población y puede causar cáncer de piel, pulmones, etc., así como favorecer el desarrollo de enfermedades cardiovasculares, neurotonales y diabetes (Choong et al., 2007; Guan et al., 2012; OMS, 2014). Asociados a estos metales también se pueden encontrar otros elementos potencialmente contaminantes como son el $\mathrm{Pb}, \mathrm{Zn}, \mathrm{Cu}$ y $\mathrm{Cd}$, los cuales cuando alcanzan elevadas concentraciones pueden llegar a generan un riesgo para el medio ambiente y para la población expuesta.

Por lo tanto, los objetivos de este trabajo fueron tanto determinar la concentración de $\mathrm{Hg}, \mathrm{As}, \mathrm{Cu}, \mathrm{Cd}, \mathrm{Pb}$ y $\mathrm{Zn}$ en suelos y sedimentos de ríos y lagos en diferentes distritos mineros de Bolivia, así como identificar el origen geológico o antropogénico de los mismos.

\section{Material y Métodos}

\section{Zona de estudio, diseño experimental y muestreo}

La zona de estudio está ubicada en el área protegida de "Apolobamba", $68^{\circ} 30^{\prime}-69^{\circ} 20^{\prime}$ oeste y $14^{\circ} 40^{\prime}-15^{\circ} 10$ sur, provincia de La Paz, Bolivia, donde se asientan alrededor de 100 cooperativas mineras, todas ellas ubicadas en llanuras aluviales de la meseta boliviana andina. Para este estudio, se seleccionaron tres distritos mineros de oro afectados por la contaminación de metales y metaloides (Campo, 2003), los cuales fueron: distrito minero de Sunchullí-Viscachani, distrito minero de Katantica y distrito minero Sural (Figura 1).

En el distrito minero de Sunchullí-Viscachani, se tomaron 2 perfiles de suelo localizados a $6 \mathrm{~km}$ al noroeste del lago Sunchullí y a $18 \mathrm{~km}$ de la mina principal, en el lado norte de la cordillera (Figura 1), estos perfiles fueron usados para evaluar si había deposición atmosférica de metales $(\mathrm{Pb}, \mathrm{Cu}, \mathrm{Zn}, \mathrm{Cd}, \mathrm{Hg})$ y As a larga distancia; siendo un Histosol fíbrico (Perfil I) compuesto por tres horizontes: H1 $(0-12 \mathrm{~cm}), \mathrm{H} 2$ (12$24 \mathrm{~cm}$ ) y $\mathrm{R}(>24 \mathrm{~cm}$ ), y un Cambisol Háplico (Perfil II) compuesto por cuatro horizontes; H1 (0-9 cm), Ah (9$24 \mathrm{~cm}), \mathrm{Bw}(24-40 \mathrm{~cm})$ y C / R (>40 cm) (WRB 2006). De igual modo se tomaron dos Regosoles Háplicos (Eútricos) (Perfil III y IV) ubicados a menos de $500 \mathrm{~m}$ de la mina principal; el Perfil III compuesto por cuatro horizontes: $\mathrm{H} 1(0-11 \mathrm{~cm}), \mathrm{AC}(11-22 \mathrm{~cm}), \mathrm{Bw}(22-40 \mathrm{~cm})$ y $R(>40 \mathrm{~cm})$ y el Perfil IV compuesto por cuatro horizontes: Ah $(0-12 \mathrm{~cm}), \mathrm{C} 1(12-32 \mathrm{~cm}), 2 \mathrm{C} 2(32-54 \mathrm{~cm})$ y 2 C3 (> $54 \mathrm{~cm}$ ) (Acosta et al., 2011). Finamente, para evaluar la acumulación de metales $(\mathrm{Pb}, \mathrm{Cu}, \mathrm{Zn}, \mathrm{Cd}$, $\mathrm{Hg}$ ) y As en los cuerpos de agua, se tomaron siete muestras de sedimentos: tres en el lago Sunchullí, dos en el lago Viscachani y cuatro en el río Sunchullí (dos antes y dos después de un punto de descarga).

El distrito minero de Katantica, ubicado en la parte norte del área minera de Apolobamba, cerca del del distrito minero Sural, incluye tres ríos, Pelechuco, Turcos y Rayo Rojo. La actividad minera en esta área, al igual que en las otras, se asocia principalmente a la extracción de oro, pero en este caso, la extracción se realiza a partir de dos materiales diferentes: cuarzo gris aurífero y depósito aluvial procedente aguas arriba y ubicado en la parte baja de los ríos mencionados anteriormente. En esta zona se muestreó un Leptosol lítico (Perfil I), compuesto por dos horizontes: Ah (0$42 \mathrm{~cm})$ y $\mathrm{C}(>42 \mathrm{~cm}$ ) y ubicado a $1 \mathrm{~km}$ de la actividad minera (Acosta et al., 2011). Adicionalmente, se tomaron dos muestras de sedimento en el lago Katantica, dos en el río Pelechuco (una antes y una después de los puntos de descarga), una en el río Rayo Rojo y otra en el ríoTurcos.

La zona minera de oro más pequeña incluida en este estudio, fue el distrito minero Sural, el cual se encuentra en la parte noroeste de Apolobamba. En esta región, las principales actividades mineras para la extracción de oro se realizan en los depósitos aluviales de la parte inferior del río Sural. En este caso se tonaron dos perfiles. El primero de ellos (Perfil I, Leptosol Lítico) está compuesto por dos horizontes: Ah $(0-42 \mathrm{~cm})$ y C (> $42 \mathrm{~cm})$ mientras que el Perfil II (Histosol Fíbrico) estaba compuesto por dos horizontes: Ah $(0-42 \mathrm{~cm})$ y $\mathrm{C}(>42 \mathrm{~cm})$, ambos se tomaron a 5 

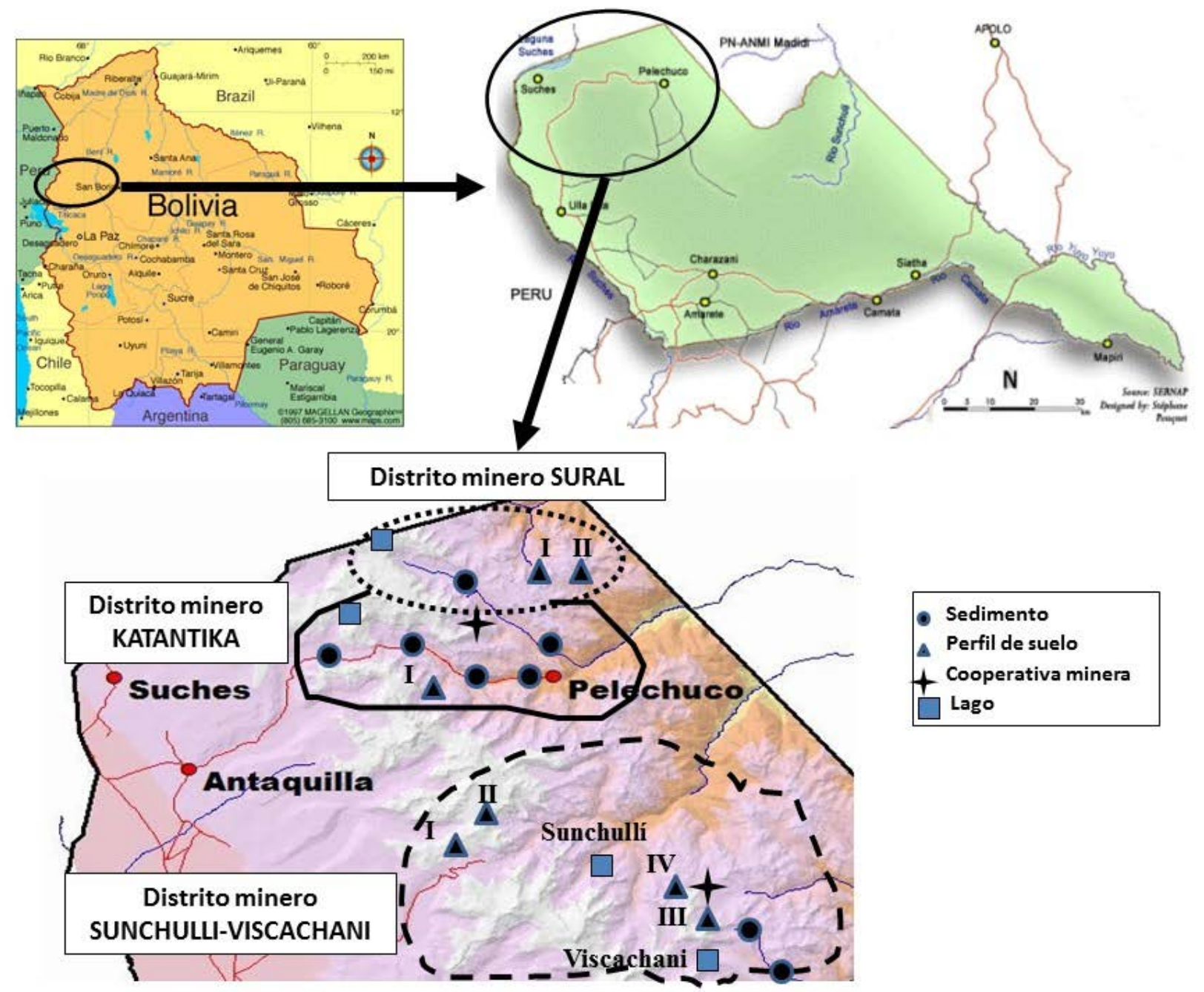

Fig_1.tiff

$\mathrm{km}$ al sur del lago Sural y a $1 \mathrm{~km}$ al este del río Sural (Acosta et al., 2011). De igual modo, se recogieron dos muestras de sedimentos en el lago Sural.

Las muestras de suelo se tomaron teniendo la precaución de evitar la contaminación entre horizontes, limpiando la pala de muestreo antes de usarla para cada muestra. Las muestras de sedimentos se tomaron con una separación de la orilla de 2 metros. Las muestras de suelo y sedimento fueron almacenadas en bolsas de polietileno para su transporte.

\section{Análisis de laboratorio}

Las muestras de suelo y sedimento se secaron a $30^{\circ} \mathrm{C}$ durante $24 \mathrm{~h}$ en estufa de aire forzado, se tamizaron con un tamiz de $2 \mathrm{~mm}$ y una fracción fue molida con mortero mecánico de ágata (Retsch RM 100) durante 10-15 minutos antes de los análisis.

La caracterización físico-química de los suelos y sedimentos consistió en la determinación de pH (Peech 1965), carbono orgánico (Anne 1945, Duchafour 1970), nitrógeno total (Duchafour 1970), conductividad eléctrica (Bower y Wilcox 1965), distribución del tamaño de partículas (FAO-ISRIC, 2006), y capacidad de intercambio catiónico (Chapman 1965).

Para la determinación de $\mathrm{Hg}$ se usó un analizador avanzado de $\mathrm{Hg}$ (AMA-254, LECO) con un límite de detección de $0,5 \mathrm{mg} \mathrm{kg}^{-1}$, este equipo utiliza muestras sólidas, por lo que no es necesaria la digestión de las muestras. Se utilizaron blancos y materiales de referencia para el control de calidad durante el análisis. Para la determinación de $\mathrm{Pb}, \mathrm{Cu}, \mathrm{As}, \mathrm{Zn}$ y Cd, las muestras fueron digeridas con ácido nítrico-perclórico siguiendo el método sugerido por Risser y Baker (1990), y estos elementos medidos mediante espectrofotómetro de absorción atómica (modelo UNICAM SOLAAR 969/989). Se utilizaron suelos de referencia (SO-3 y SO-4) procedentes del Canadian Certified Reference Materials Project para el control de calidad durante los análisis (Bowman et al., 1979).

Con el fin de evaluar el grado de contaminación, se consideraron los niveles de referencia propuestos 
en Bélgica para $\mathrm{Cd}\left(0,8 \mathrm{mg} \mathrm{kg}^{-1}\right), \mathrm{Cu}\left(17 \mathrm{mg} \mathrm{kg}^{-1}\right), \mathrm{Pb}$ $\left(40 \mathrm{mg} \mathrm{kg}^{-1}\right)$ y $\mathrm{Zn}\left(62 \mathrm{mg} \mathrm{kg}^{-1}\right)$ (BWRHABTGG , 1995), mientras que en el caso de $\mathrm{Hg}$ y As se consideran los valores de referencia de Holanda $\left(0,3 \mathrm{mg} \mathrm{Hg} \mathrm{kg}^{-1}\right.$ y 19 mg As $\mathrm{kg}^{-1}$ ) (NMHPPE, 1994).

\section{Resultados y discusión}

\section{Propiedades físico-químicas de los suelos}

Los resultados de las propiedades físico-químicas de suelo de cada horizonte de los distritos mineros de Sunchullí-Viscachani, Katantika y Sural se presentan en la Tabla 1. El pH de los suelos varió entre 4,55 y 6,91 en el Distrito minero de Sunchulli-Viscachani, entre 4,75 y 6,40 en Katantica y entre 4,04 y 5,09 en el distrito minero Sural, y como era de esperar no observó presencia de carbonatos en ninguno de los perfiles de suelo evaluados. Por lo tanto, los suelos en los tres distritos mineros pueden clasificarse como fuertemente ácidos y neutros (Soil Survey Staff, 2006); los cuales favorecen la solubilización de los metales que pudieran contener, incrementando su movilidad (Kabata-Pendias y Pendias, 2010). Por su parte, la conductividad eléctrica (C.E.), que es u reflejo directo de la salinidad, osciló entre 50 y $730 \mathrm{mS} \mathrm{cm}^{-1}$ en SunchuIli-Viscachani, entre 40 a $150 \mathrm{mS} \mathrm{cm}-1$ en Katantica y entre 110 a $370 \mathrm{mS} \mathrm{cm}-1$ en el distrito minero Sural, por lo tanto, los suelos pueden clasificarse como no salinos (USDA, 2003).
Los contenidos de COT, NT y CIC siguieron el mismo patrón, con los valores más altos en el horizonte superior del perfil en los tres distritos mineros, siendo altos en comparación con los resultados encontrados por otros autores en pastizales de altura (Zemmrich et al. 2010, Podwojewski et al., 2011), lo cual está estrechamente relacionado con la alta cobertura vegetal observada en estas zonas. No obstante, la disminución observada en el contenido de COT y NT en la zona ubicada cerca de los lugares de extracción minera (perfil III y IV), podría dar lugar a la desaparición de la cobertura vegetal, lo cual podría afectar a la estabilidad estructural del suelo y, por lo tanto, promover los procesos erosivos y degradación.

\section{Propiedades físico-químicas de los sedimentos de las lagunas y ríos}

Las propiedades físico-químicas de los sedimentos muestreados en los lagos Sunchulli, Viscachani, Katantika y Sural se muestran en la Tabla 2. Los valores de $\mathrm{pH}$ de los sedimentos de los lagos fueron neutros $(6,3-7,1)$ en Sunchulli, moderadamente ácidos $(5,7)$ en Viscachani, ligeramente ácidos (6,1-6,9) en Sural y de fuertemente ácidos a neutros $(5,18-6,49)$ en Katantica (Soils Survey Division Staff, 1993); donde nuevamente no se observaron carbonatos (Cobertera, 1993). Desde el punto de vista de la salinidad, los sedimentos muestreados no eran salinos (Soils Survey Division Staff, 1993), ya que el valor más elevado fue registrado en la laguna Viscachani, con un valor de $560 \mathrm{~ms}$

\begin{tabular}{|c|c|c|c|c|c|c|}
\hline Perfiles de suelo & pH & 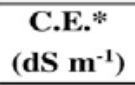 & $\begin{array}{l}\text { C.O.T. } \\
\left(\mathrm{g} \mathrm{kg}^{-1}\right)\end{array}$ & $\begin{array}{c}\text { C.I.C. } \\
\left(\mathrm{cmol}_{+} \mathrm{kg}^{-1}\right)\end{array}$ & $\begin{array}{c}\text { N.T. } \\
\left(\mathrm{g} \mathrm{kg}^{-1}\right)\end{array}$ & $\begin{array}{l}\text { Arcilla } \\
\left(\mathrm{g} \mathrm{kg}^{-1}\right)\end{array}$ \\
\hline \multicolumn{7}{|c|}{ Distrito minero Sunchulli-Viscachani } \\
\hline Perfil I - H1 $(0-12 \mathrm{~cm})$ & 5,55 & 0,20 & 96,6 & 30,57 & 7,7 & 176,8 \\
\hline Perfil I - H2 (12-24 cm) & 5,77 & 0,21 & 45,4 & 11,90 & 3,4 & 112,0 \\
\hline Perfil II - H $(0-9 \mathrm{~cm})$ & 6,91 & 0,25 & 66,2 & 15,26 & 3,8 & 169,3 \\
\hline Perfil II - Ah $(9-24 \mathrm{~cm})$ & 5,41 & 0,06 & 27,6 & 9,80 & 2,6 & 146,5 \\
\hline Perfil II - Bw (24-40 cm) & 6,61 & 0,05 & 16,8 & 7,43 & 1,5 & 81,5 \\
\hline Perfil II - C/R (>40 cm) & 5,44 & 0,19 & 19,2 & 5,76 & 1,4 & 89,7 \\
\hline Perfil III - Ah $(0-11 \mathrm{~cm})$ & 5,13 & 0,16 & 39,2 & 12,36 & 3,4 & 156,0 \\
\hline Perfil III - A/C $(11-22 \mathrm{~cm})$ & 5,27 & 0,12 & 27,0 & 11,01 & 2,1 & 127,3 \\
\hline Perfil III - C (22-40 cm) & 5,88 & 0,06 & 5,8 & 3,63 & 0,5 & 79,6 \\
\hline Perfil IV - Ah (0-12 cm) & 5,09 & 0,73 & 41,0 & 14,41 & 5,9 & 208,5 \\
\hline Perfil IV - C1 $(12-32 \mathrm{~cm})$ & 4,55 & 0,51 & 30,1 & 15,78 & 3,6 & 259,5 \\
\hline Perfil IV $-2 \mathrm{C} 2(32-54 \mathrm{~cm})$ & 5,31 & 0,17 & 20,8 & 12,28 & 1,6 & 161,9 \\
\hline Perfil IV $-2 \mathrm{C} 3(>54 \mathrm{~cm})$ & 5,12 & 0,32 & 16,3 & 11,64 & 1,5 & 125,1 \\
\hline \multicolumn{7}{|l|}{ Distrito minero Katantica } \\
\hline Perfil I - Ah & 4,75 & 0,15 & 75,2 & 24,68 & 4,3 & 109,2 \\
\hline Perfil I - C & 6,40 & 0,04 & 06,6 & 4,14 & 0,5 & 55,0 \\
\hline \multicolumn{7}{|l|}{ Distrito minero Sural } \\
\hline Perfil I - A & 5,09 & 0,37 & 43,7 & 15,21 & 4,6 & 95,7 \\
\hline Perfil II - H & 4,04 & 0,11 & 59,8 & 61,01 & 17,0 & 150,8 \\
\hline
\end{tabular}

Tabla 1. Propiedades de suelo en los distritos mineros Sunchulli-Viscachani, Katantika y Sural.

Table 1. Soil properties in the mining district of Sunchulli-Viscachani, Katantika and Sural. 


\begin{tabular}{|c|c|c|c|c|c|c|}
\hline Sedimentos lacustres & $\mathrm{pH}$ & $\begin{array}{c}\text { C.E.* } \\
\left(\mathrm{dS} \mathrm{m}^{-1}\right)\end{array}$ & $\begin{array}{l}\text { C.O.T. } \\
\left(\mathrm{g} \mathrm{kg}^{-1}\right)\end{array}$ & $\begin{array}{c}\text { C.I.C. } \\
\left(\mathrm{cmol}_{+} \mathbf{k g}^{-1}\right)\end{array}$ & $\begin{array}{c}\text { N.T. } \\
\left(\mathrm{g} \mathrm{kg}^{-1}\right)\end{array}$ & $\begin{array}{l}\text { Arcilla } \\
\left(\mathrm{g} \mathrm{kg}^{-1}\right)\end{array}$ \\
\hline \multicolumn{7}{|l|}{ Laguna Sunchullí } \\
\hline Sedimento (1) & 7,19 & 0,41 & 10,6 & 1,18 & 0,5 & 12,9 \\
\hline Sedimento (2) & 6,31 & 0,11 & 18,3 & 7,17 & 2,7 & 99,4 \\
\hline Sedimento (3) & 7,02 & 0,21 & 11,9 & 3,29 & 1,2 & 76,8 \\
\hline \multicolumn{7}{|l|}{ Laguna Viscachani } \\
\hline Sedimento (1) & 5,74 & 0,56 & 218,3 & 34,74 & 16,7 & 205,1 \\
\hline Sedimento (2) & 5,77 & 0,04 & 89,5 & 42,56 & 9,0 & 277,2 \\
\hline \multicolumn{7}{|l|}{ Laguna Sural } \\
\hline Sedimento (1) & 6,94 & 0,17 & 11,8 & 5,56 & 1,0 & 127,1 \\
\hline Sedimento (2) & 6,18 & 0,14 & 62,9 & 11,42 & 4,4 & 104,9 \\
\hline \multicolumn{7}{|l|}{ Laguna Katantica } \\
\hline Sedimento (1) & 6,48 & 0,32 & 16,7 & 4,56 & 1,7 & 68,2 \\
\hline Sedimento (2) & 5,18 & 0,67 & 87,6 & 31,35 & 8,1 & 138,7 \\
\hline
\end{tabular}

*C.E.: conductividad eléctrica; C.O.T.: carbono orgánico total; N.T.: nitrógeno total; C.I.C.: capacidad de intercambio catiónico.

Tabla 2. Propiedades de los sedimentos de las lagunas Sunchulli, Viscachani, Katantika y Sural.

Table 2. Sediment properties in the lakes of Sunchulli, Viscachani, Katantika and Sural.

$\mathrm{cm}^{-1}$. Por su parte, las concentraciones de COT y NT de los sedimentos de Viscachani fueron las más altas, mientras que en la laguna Sunchilli fueron las más bajas, lo que podría explicarse debido al aporte de materia orgánica por parte de la vegetación que crecía en las orillas del lago, mucho más abundante en la laguna Viscachani. Nuevamente, los mayores valores de CEC fueron encontradas en la laguna Viscachani, lo que está estrechamente relacionado con las altas concentraciones de COT y el contenido de arcilla (Honorato, 2000).

El pH de los sedimentos de los ríos varió entre 4,85 (muy fuertemente ácido en el río Sunchulli) y 7,41 (Iigeramente alcalino, en el río Pelechuco) (Soils Survey Division Staff, 1993) (Tabla 3). Por su parte, atendiendo a los valores de conductividad eléctrica, los sedimentos de todos los ríos eran no salinos (Soils Survey Division Staff, 1993). Finalmente, las concentraciones de CIC, COT y NT de los sedimentos de los ríos muestrea- dos fueron más bajas que las encontradas en los sedimentos de la laguna, lo cual es probablemente debido a que las aguas en movimiento de los ríos arrastran la materia orgánica no favoreciendo su deposición en el lecho de los mismos, aspecto que sí se ve favorecido en los lagos, donde las aguas se mantienen estacadas por largos periodos de tiempo, lo que favorece la deposición y descomposición de esta materia orgánica.

\section{Concentraciones de metales pesados y As en los suelos}

Las concentraciones de $\mathrm{Hg}$ y $\mathrm{As}$ en el horizonte superficial de los cuatro perfiles en el Distrito Minero de Sunchullí-Viscachani fueron 0,$38 ; 2,58 ; 9,94$ y 2,96 mg Hg kg-1; y 6,$85 ; 14,95 ; 18,49 ; 20,45$ mg As kg-1 $^{-1}$, respectivamente. Por su parte, las concentraciones en el horizonte más profundo fueron 0,$09 ; 0,34 ; 1,27 \mathrm{y}$ $0,23 \mathrm{mg} \mathrm{Hg} \mathrm{kg}^{-1}$ y 9,$24 ; 8,84 ; 8,67$ y $11,03 \mathrm{mg} \mathrm{As} \mathrm{kg}^{-1}$, respectivamente (Tabla 4). Estos resultados sugieren

\begin{tabular}{|c|c|c|c|c|c|c|}
\hline Sedimentos de río & pH & $\begin{array}{c}\text { C.E. } \\
\left(\mathrm{dS} \mathrm{m}^{-1}\right)\end{array}$ & $\begin{array}{l}\text { C.O.T. } \\
\left(\mathrm{g} \mathrm{kg}^{-1}\right)\end{array}$ & $\begin{array}{c}\text { C.I.C. } \\
\left(\mathrm{cmol}_{+} \mathbf{k g}^{-1}\right)\end{array}$ & $\begin{array}{c}\text { N.T. } \\
\left(\mathrm{g} \mathrm{kg}^{-1}\right)\end{array}$ & $\begin{array}{l}\text { Arcilla } \\
\left(\mathrm{g} \mathrm{kg}^{-1}\right)\end{array}$ \\
\hline \multicolumn{7}{|l|}{ Río Sunchullí } \\
\hline Sedimento (1) antes del vertido & 6,34 & 0,226 & 11,58 & 4,18 & 1,18 & 40,84 \\
\hline Sedimento (2) después del vertido & 4,85 & 1,181 & 16,55 & 2,99 & 0,91 & 52,82 \\
\hline \multicolumn{7}{|l|}{ Río Pelechuco } \\
\hline Sedimento (1) antes del vertido & 6,35 & 0,07 & 8,4 & 1,99 & 1,0 & 19,0 \\
\hline Sedimento (2) después del vertido & 7,41 & 0,27 & 33,1 & 4,84 & 3,6 & 418,7 \\
\hline \multicolumn{7}{|l|}{ Río Turcos } \\
\hline Sedimento (1) & 7,27 & 0,29 & 6,22 & 1,11 & 0,34 & 12,52 \\
\hline \multicolumn{7}{|l|}{ Río Rayo Rojo } \\
\hline Sedimento (1) & 7,03 & 0,55 & 6,29 & 0,96 & 0,35 & 9,98 \\
\hline
\end{tabular}

Tabla 3. Propiedades de los sedimentos de los riegos Sunchulli, Pelechuco, Turcos y Rayo Rojo.

Table 3. Sediment properties in the rivers of Sunchulli, Pelechuco, Turcos and Rayo Rojo. 


\begin{tabular}{|c|c|c|c|c|c|c|}
\hline Perfiles de suelo & $\begin{array}{c}\text { Plomo } \\
\left(\mathrm{mg} \mathrm{kg}^{-1}\right)\end{array}$ & $\begin{array}{c}\text { Cobre } \\
\left(\mathrm{mg} \mathrm{kg}^{-1}\right)\end{array}$ & $\begin{array}{c}\text { Cinc } \\
\left(\mathrm{mg} \mathrm{kg}^{-1}\right)\end{array}$ & $\begin{array}{l}\text { Cadmio } \\
\left(\mathrm{mg} \mathrm{kg}^{-1}\right)\end{array}$ & $\begin{array}{c}\text { Arsénico } \\
\left(\mathrm{mg} \mathrm{kg}^{-1}\right)\end{array}$ & $\begin{array}{c}\text { Mercurio } \\
\left(\mathrm{mg} \mathrm{kg}^{-1}\right)\end{array}$ \\
\hline \multicolumn{7}{|c|}{ Distrito Sunchullí-Viscachani } \\
\hline Perfil I - H1 (0-12 cm) & 5,6 & 12,7 & 59,1 & 0,26 & 6,85 & 0,38 \\
\hline Perfil I - H2 (12-24 cm) & 4,2 & 9,4 & 66,5 & 0,01 & 9,24 & 0,09 \\
\hline Perfil II - H $(0-9 \mathrm{~cm})$ & 27,8 & 38,6 & 92,7 & 0,02 & 14,95 & 2,58 \\
\hline Perfil II - Ah $(9-24 \mathrm{~cm})$ & 9,3 & 29,1 & 100,4 & 2,43 & 9,96 & 0,05 \\
\hline Perfil II - Bw $(24-40 \mathrm{~cm})$ & 18,2 & 20,3 & 91,9 & 0,25 & 4,89 & 0,08 \\
\hline Perfil II - C/R (>40 cm) & 29,5 & 26,8 & 140,5 & 0,25 & 8,84 & 0,34 \\
\hline Perfil III - Ah $(0-11 \mathrm{~cm})$ & 23,8 & 17,4 & 84,3 & 2,84 & 18,49 & 9,94 \\
\hline Perfil III - A/C (11-22 cm) & 26,2 & 22,1 & 94,8 & 2,04 & 8,50 & 0,05 \\
\hline Perfil III - C (22-40 cm) & 37,1 & 32,4 & 82,8 & 0,01 & 8,67 & 1,27 \\
\hline Perfil IV - Ah $(0-12 \mathrm{~cm})$ & 33,1 & 23,0 & 81,7 & 1,68 & 20,45 & 2,96 \\
\hline Perfil IV - Cl (12-32 cm) & 35,8 & 24,9 & 101,9 & 1,00 & 16,07 & 1,97 \\
\hline Perfil IV - 2C2 $(32-54 \mathrm{~cm})$ & 29,6 & 25,0 & 71,3 & 1,27 & 14,30 & 0,22 \\
\hline Perfil IV - 2C3 (>54 cm) & 24,5 & 27,0 & 71,1 & 2,04 & 11,03 & 0,23 \\
\hline \multicolumn{7}{|l|}{ Distrito Minero Katantica } \\
\hline Perfil I - Ah & 15,6 & 17,7 & 140,3 & 1,88 & 10,99 & 0,08 \\
\hline Perfil I - C & 8,6 & 18,3 & 143,5 & 3,49 & 4,13 & 0,02 \\
\hline \multicolumn{7}{|l|}{ Distrito Minero Sural } \\
\hline Perfil I - A & 18,3 & 19,6 & 145,7 & 4,66 & 5,53 & 0,06 \\
\hline Perfil II - H & 13,5 & 6,4 & 15,5 & 5,34 & 12,34 & 0,07 \\
\hline Legislación* & 40 & 17 & 62 & 0,8 & 19 & 0,3 \\
\hline
\end{tabular}

Tabla 4. Metales y As en los distritos mineros Sunchullí-Viscachani, Katantika y Sural.

Table 4. Metals and As in the mining district of Sunchulli-Viscachani, Katantika and Sural.

que la deposición atmosférica de $\mathrm{Hg}$ y As podría estar teniendo lugar en este Distrito ya que en superficie la concentración en todos los casos, a excepción de As para el perfil l, es muy superior que en profundidad. Algunos estudios han demostrado que, en muchas áreas tropicales, el $\mathrm{Hg}$ suele ser alto en los suelos, probablemente debido tanto a la deposición atmosférica de $\mathrm{Hg}$ durante años (Lechler et al., 2000) como a la caída y acumulación de hojas con contenidos relativamente altos de $\mathrm{Hg}$ (Ericksen et al., 2003)

Probablemente, las concentraciones observadas en el Perfil I representan la acumulación natural de $\mathrm{Hg}$ y As en los horizontes de suelo. Por el contrario, los perfiles II, III y IV mostraron una concentración mucho mayor en el horizonte superficial que en el horizonte de profundidad, lo que indicaría una deposición atmosférica activa de estos dos elementos. Mulholland et al. (2012) demostró que el mercurio se depositaba en su forma metálica en los suelos procedente de la extracción de oro por amalgamación artesanal. De igual modo, se ha demostrado que el $\mathrm{Hg}$ se puede transportar en el aire largas distancias adherido a pequeñas partículas (Peñaloza y Reinhardt, 2000). Estas partículas podrían alcanzar los suelos agrícolas y los pastos cercanos a las áreas de explotación minera causando riesgos tanto para el medio ambiente, como para la población expuesta.

Los resultados (Tabla 4) muestran que las concentraciones de los metales pesados estudiados, principalmente $\mathrm{Pb}, \mathrm{Cu}$ y $\mathrm{Zn}$, también son más altas en el
Perfil II que en el Perfil I. De hecho, las concentraciones de $\mathrm{Pb}$ en los horizontes superficiales alcanzan los $5,6 \mathrm{mg} \mathrm{kg}^{-1}$ en el Perfil I y los $28 \mathrm{mg} \mathrm{kg}^{-1}$ en el Perfil II, sin embargo, en este caso esta diferencia puede que sea debida a la presencia natural de $\mathrm{Pb}$. Existe una tendencia natural a la acumulación de $\mathrm{Pb}$ en las series félsicas de las rocas magmáticas, así como en sedimentos arcillosos, como es el caso que nos ocupa, donde los contenidos de este elemento varían entre 10 y $40 \mathrm{mg} \mathrm{kg}^{-1}$ (Kabata-Pendias y Pendias 2010), concentraciones en el rango de las determinadas en este estudio. Por su parte, la concentración de $\mathrm{Pb}$ en el horizonte $\mathrm{C} / \mathrm{R}$ en el perfil II es similar a la concentración de $\mathrm{Pb}$ en el horizonte superficial, lo que apoya la hipótesis del enriquecimiento natural de este elemento. De igual modo, las concentraciones de $\mathrm{Pb}, \mathrm{Cu}$ y $\mathrm{Zn}$ son similares en los Perfiles III y IV y muestran un aumento con la profundidad de $\mathrm{Pb}$ y $\mathrm{Cu}$ (Tabla 4), lo que indica que posiblemente el origen geogénico es la fuente principal de estos metales.

Por su parte, en los Distritos Mineros Katantica y Sural las concentraciones de $\mathrm{Hg}, \mathrm{As}, \mathrm{Pb}$ y $\mathrm{Cu}$ fueron inferiores a las encontradas en el Distrito Minero Sunchullí-Viscachani (Tabla 4), de igual modo, las concentraciones de estos elementos fueron inferiores a las concentraciones de referencia según las legislaciones utilizadas, por lo tanto, se podría decir que no existe enriquecimiento antropogénico de $\mathrm{Hg}, \mathrm{As}, \mathrm{Pb}$ y $\mathrm{Cu}$ en estos perfiles. Sin embargo, las concentraciones de $\mathrm{Cd}$ y $\mathrm{Zn}$ fueron más altas que en el Distrito Minero de 
Sunchullí-Viscachani y superaron ampliamente los niveles umbrales $\left(0,8\right.$ y $62 \mathrm{mg} \mathrm{kg}^{-1}$ para $\mathrm{Cd}$ y $\mathrm{Zn}$, respectivamente), lo que indica un mayor riesgo ambiental, especialmente en caso del $\mathrm{Cd}$, no obstante, debido a que las concentraciones más altas fueron encontradas en los horizontes de profundidad, posiblemente su origen sea geogénico.

\section{Concentraciones de metales pesados y As en los sedi- mentos de las lagunas y ríos}

Las concentraciones de $\mathrm{Pb}, \mathrm{Cu}, \mathrm{Cd}, \mathrm{Zn}, \mathrm{Hg}$ y As en los sedimentos de las lagunas muestreadas se presentan en la Tabla 5. De acuerdo con la legislación utilizada, la mayoría de los sedimentos de las lagunas Sunchullí y Viscachani podrían clasificarse como contaminados por $\mathrm{Hg}$, As, Cd, Zn y Cu (Tabla 4 y 5), lo que podría ocasionar un riesgo para los ecosistemas de estas lagunas, así como la población que estuviera expuesta a las mismas. Probablemente, las concentraciones de $\mathrm{Hg}$ y As presentes en los sedimentos de estas lagunas procedan de los procesos de amalgamación realizados en las inmediaciones de las mismas, cuyos residuos podrían haber sido vertidos directamente a las mismas debido a su proximidad. De hecho, la concentración más alta de $\mathrm{Hg}\left(23,7 \mathrm{mg} \mathrm{kg}^{-1}\right)$ se registró en el sedimento (2) de la laguna Viscachani, cuya muestra fue tomada cerca de un área donde existían evidencias de haberse llevado a cabo procesos de amalgamación. Esta concentración fue similar a la encontrada en los sedimentos del río Madeira (Brasil) $\left(19,83 \mathrm{mg} \mathrm{kg}^{-1}\right)$ también afectado por este tipo de minería (Pfeiffer et al., 1991). De igual modo, Cd y Zn presentan una mayor concentración en el sedimento (2) de la laguna Viscachani, lo que indicaría una contribución antropogénica de estos dos elementos probablemente debido al proceso de amalgamación. Por el contrario, $\mathrm{Pb}$ y $\mathrm{Cu}$ se comportan de forma inversa a los metales anteriores por lo que su procedencia po- dría ser de carácter geogénico.

En lo que respecta al lago Katantica (Tabla 5), no se encontró enriquecimiento por $\mathrm{Pb}, \mathrm{Cu}$ y $\mathrm{As}$ en sus sedimentos, presentando concentraciones inferiores a los niveles propuestos por las legislaciones utilizadas (Tabla 4). Sin embargo, el sedimento (1), ubicado junto a las operaciones mineras, presentó una concentración de $2,24 \mathrm{mg} \mathrm{Hg} \mathrm{kg}^{-1}$ mostrando cierto enriquecimiento por Hg (Tabla 5). Del mismo modo, se encontraron altas concentraciones de $\mathrm{Zn}$ y $\mathrm{Cd}$ en ambos sedimentos tomados en esta laguna, lo que indica que los sedimentos son sumideros de estos metales. Por lo que en este caso, se podría atribuir un origen geológico a las concentraciones de $\mathrm{Pb}, \mathrm{Cu}$ y $\mathrm{As}$, mientras que sería antropogénico las de $\mathrm{Hg}, \mathrm{Zn}$ y $\mathrm{Cd}$.

Finalmente, las concentraciones de $\mathrm{Hg}, \mathrm{Pb}$ y $\mathrm{Cd}$ en los sedimentos de la laguna Sural fueron bajos (Tabla 5), todos ellos inferiores a los niveles propuestos por la legislación utilizada, con niveles que indicarían un origen geogénico de los mismos. Por el contrario, las concentraciones de $\mathrm{Zn}, \mathrm{Cu}$ y As excedieron esos niveles, siendo significativamente más elevadas que en las lagunas Viscachani y Katantica, lo que indica un enriquecimiento de estos elementos en los sedimentos, probablemente de origen antrópico y, por lo tanto, un riesgo potencial para el ecosistema de la laguna.

Las concentraciones de metales y As en los sedimentos de los ríos muestreados se muestran en la Tabla 6. En el caso del río Sunchullí, las concentraciones de $\mathrm{Pb}$ y $\mathrm{Hg}$ en los sedimentos muestreados antes y después de los puntos de vertido fueron de $15,7 \mathrm{mg}$ $\mathrm{Pb} \mathrm{kg}-1,4,1 \mathrm{mg} \mathrm{Hg} \mathrm{kg}^{-1}$ y $99 \mathrm{mg} \mathrm{Pb} \mathrm{kg}{ }^{-1}, 103 \mathrm{mg} \mathrm{Hg}$ $\mathrm{kg}^{-1}$, respectivamente, confirmando el significativo incremento de $\mathrm{Hg}$ y $\mathrm{Pb}$ procedente de las zonas de extracción y procesado de mercurio (Tabla 6), generando impactos negativos tanto al medio ambiente como a la salud de la población expuesta. De igual modo, las concentraciones de $\mathrm{Cu}$ y As se incrementan sig-

\begin{tabular}{|c|c|c|c|c|c|c|}
\hline Sedimentos lacustres & $\begin{array}{c}\text { Plomo } \\
\left(\mathrm{mg} \mathrm{kg}^{-1}\right)\end{array}$ & $\begin{array}{c}\text { Cobre } \\
\left(\mathrm{mg} \mathrm{kg}^{-1}\right)\end{array}$ & $\begin{array}{c}\text { Cinc } \\
\left(\mathrm{mg} \mathrm{kg}^{-1}\right)\end{array}$ & $\begin{array}{l}\text { Cadmio } \\
\left(\mathrm{mg} \mathrm{kg}^{-1}\right)\end{array}$ & $\begin{array}{c}\text { Arsénico } \\
\left(\mathrm{mg} \mathrm{kg}^{-1}\right)\end{array}$ & $\begin{array}{c}\text { Mercurio } \\
\left(\mathrm{mg} \mathrm{kg}^{-1}\right)\end{array}$ \\
\hline \multicolumn{7}{|l|}{ Laguna Sunchullí } \\
\hline Sedimento (1) & 6,2 & 21,9 & 47,3 & 1,47 & 86,75 & 12,3 \\
\hline Sedimento (2) & 0,6 & 72,1 & 106,5 & 0,32 & 46,03 & 0,1 \\
\hline Sedimento (3) & 1,3 & 29,8 & 85,1 & 2,45 & 33,57 & 4,14 \\
\hline \multicolumn{7}{|l|}{ Laguna Viscachani } \\
\hline Sedimento (1) & 38,6 & 27,3 & 39,9 & 0,93 & 10,64 & 11,7 \\
\hline Sedimento (2) & 23,9 & 19,5 & 99,3 & 2,52 & 16,93 & 23,7 \\
\hline \multicolumn{7}{|l|}{ Laguna Sural } \\
\hline Sedimento (1) & 5,2 & 41,0 & 136,4 & 0,10 & 28,16 & 0,07 \\
\hline Sedimento (2) & 14,2 & 31,7 & 179,1 & 0,05 & 51,04 & 0,23 \\
\hline \multicolumn{7}{|l|}{ Laguna Katantica } \\
\hline Sedimento (1) & 13,2 & 8,5 & 70,4 & 4,88 & 17,77 & 2,24 \\
\hline Sedimento (2) & 26,5 & 13,3 & 95,5 & 3,48 & 18,04 & 0,20 \\
\hline
\end{tabular}

Tabla 5. Metales and As en los sedimentos de los lagos Sunchullí, Viscachani, Katantica y Sural, Table 5. Metals and As in the lakes of Sunchulli, Viscachani, Katantika and Sural. 


\begin{tabular}{lcccccc}
\hline \multicolumn{1}{c}{ Sedimentos de río } & $\begin{array}{c}\text { Plomo } \\
\left(\mathbf{m g ~ k g}^{-1}\right)\end{array}$ & $\begin{array}{c}\text { Cobre } \\
\left(\mathbf{m g ~ k g}^{-1}\right)\end{array}$ & $\begin{array}{c}\text { Cinc } \\
\left(\mathbf{m g ~ k g}^{-1}\right)\end{array}$ & $\begin{array}{c}\text { Cadmio } \\
\left(\mathbf{m g ~ k g}^{-1}\right)\end{array}$ & $\begin{array}{c}\text { Arsénico } \\
\left(\mathbf{m g ~ k g}^{-1}\right)\end{array}$ & $\begin{array}{c}\text { Mercurio } \\
\left(\mathbf{m g ~ k g}^{-1}\right)\end{array}$ \\
\hline $\begin{array}{l}\text { Río Sunchullí } \\
\text { Sedimento (1) antes del vertido }\end{array}$ & 15,70 & 17,30 & 81,92 & 0,01 & 0,59 & 4,14 \\
$\begin{array}{l}\text { Sedimento (2) después del vertido } \\
\text { Río Pelechuco }\end{array}$ & 99,01 & 36,22 & 91,60 & 0,03 & 11,95 & 102 \\
$\begin{array}{l}\text { Sedimento (1) antes de vertido } \\
\text { Sedimento (2) después de vertido }\end{array}$ & 3,24 & 12,66 & 119,48 & 0,01 & 14,66 & 0,08 \\
$\begin{array}{l}\text { Río Turcos } \\
\text { Sedimento (1) }\end{array}$ & 2,28 & 22,14 & 121,53 & 0,04 & 52,26 & 6,85 \\
$\begin{array}{l}\text { Río Rayo Rojo } \\
\text { Sedimento (1) }\end{array}$ & 7,74 & 6,53 & 54,78 & 0,02 & 3,59 & 0,04 \\
& 20,81 & 15,28 & 36 & 0,02 & 53,95 & 8,55 \\
\hline
\end{tabular}

Tabla 6. Metales y As en los sedimentos de los ríos Sunchullí, Pelechuco, Turcos y Rayo Rojo. Table 6. Metals and As in the rivers of Sunchulli, Pelechuco, Turcos and Rayo Rojo.

nificativamente después del punto de vertido, lo que indica que la presencia de $\mathrm{Cu}$ y As en los sedimentos del río Sunchullí procede de los vertidos directos de la actividad minera. Por el contrario, para el caso del Zn y del Cd no se observaron incrementos tras el vertido, por lo que no se puede atribuir a este la presencia de estos metales en el río Sunchullí. Es de destacar que muchos asentamientos de pequeñas poblaciones se encuentran ubicadas aguas debajo de esta zona minera, las cuales consumen agua procedente del río Sunchullí, ya sea para fines domésticos o para riego de pequeñas plantaciones, por lo que pueden estar expuestas a riesgos para la salud por el consumo de esta agua.

En el caso del río Pelechuco, se encontró un aumento en las concentraciones de $\mathrm{Hg}$, As y $\mathrm{Cu}$ en los sedimentos después del punto de vertido en comparación con la muestra tomada antes de ese punto (Tabla 6), nuevamente la actividad minera desarrollada en las inmediaciones del río, y sus continuos vertidos al mismo, han contribuido a que los sedimentos de la parte inferior de la cuenca acumulen progresivamente concentraciones más altas de $\mathrm{Hg}$, As y Cu. Finalmente, teniendo en cuenta los niveles permitidos por la legislación, estos sedimentos estarían principalmente contaminados por $\mathrm{Hg}$ y As. Por el contrario, para el caso del $\mathrm{Pb}, \mathrm{Zn}$ y $\mathrm{Cd}$ no se observaron incrementos tras el vertido, por lo que no se puede atribuir a este la presencia de estos metales en el río Pelechuco, por lo que su origen podría ser geogénico.

Finalmente, en el río Rayo Rojo se encontraron concentraciones de $\mathrm{Pb}, \mathrm{Cu}$, As y $\mathrm{Hg}$ superiores que en el ríoTurcos, sin embargo, únicamente As y $\mathrm{Hg}$ excedieron las concentraciones propuestas por la legislación para considerarlos contaminados. En el caso de estos dos ríos, al no tener ningún punto de referencia de vertidos, para evaluar el posible origen de los metales encontrados en este río, se compararon sus concentraciones con las encontradas en el resto de ríos. Como se puede apreciar (Tabla 6), en el caso del río Turcos, las concentraciones de todos los elementos evaluados se encontrarían en rangos semejantes a al resto de ríos antes del efecto del vertido, por lo que su origen en todos los casos sería geológico. Finalmente, para el río Rayo Rojo, las concentraciones de $\mathrm{Pb}, \mathrm{Cu}, \mathrm{Zn}$ y $\mathrm{Cd}$ se podrían asemejar a las encontradas en algunos de los ríos anteriores para aquellos puntos muestreados antes de los posibles vertidos, por lo que su origen podría ser geológico, mientras que para As y $\mathrm{Hg}$ las concentraciones encontradas serían significativamente superiores por lo que su origen podría ser antropogénico.

\section{Conclusiones}

De acuerdo con las concentraciones de metales y As en los suelos estudiados, el Distrito Minero de Sunchullí-Viscachani estaría contaminado por As y $\mathrm{Hg}$, probablemente debido a la deposición atmosférica de estos elementos procedentes del proceso de amalgamación, aspecto que debería ser evaluado en detalle en estudios posteriores mediante estudios mineralógicos, mineraloquímicos y texturales. Por su parte, las concentraciones de $\mathrm{Cd}$ y $\mathrm{Zn}$ superan ampliamente los niveles permitidos en los Distritos Mineros de Katantica y Sural, por lo que también podrían estar generando un riesgo tanto para los ecosistemas de estas zonas como para la población.

En relación a los sedimentos de las lagunas estudiadas, las concentraciones de $\mathrm{Cu}, \mathrm{Cd}, \mathrm{As}, \mathrm{Zn} \mathrm{y} \mathrm{Hg}$ fueron elevadas en las lagunas Sunchullí y Viscachani, teniendo un posible origen antropogénico $\mathrm{Zn}, \mathrm{Hg}$, As, $\mathrm{Cd}$ y $\mathrm{Hg}$, mientras que la laguna Katantica mostró altas concentraciones de $\mathrm{Zn}, \mathrm{Cd}$ y $\mathrm{Hg}$ debido a los vertidos directos de la actividad minera. Por su parte, en la laguna Sural se registraron altas concentraciones de As, $\mathrm{Zn}$ y $\mathrm{Cu}$, que al igual que en los casos anteriores tenían un origen antropogénico. Los resultados mostraron que los continuos vertidos a los ríos estudiados procedentes de los procesos de tratamiento de mineral y, por lo tanto, con un origen antropogénico, incrementaron las concentraciones de $\mathrm{Cu}, \mathrm{Pb}$, As y $\mathrm{Hg}$ en los sedimentos del río Sunchullí, de $\mathrm{Hg}$, As y $\mathrm{Cu}$ en el río Pelechuco y, de As y Hg en el río Rayo Rojo. Por lo 
tanto, las altas concentraciones registradas en los sedimentos de lagunas y ríos pueden estar causando un grave problema tanto para los ecosistemas acuáticos como para la salud de la población expuesta, además, el proceso de metilación de Hg podría estar ocurriendo en los sedimentos, lo que podría representar un mayor riesgo para el medio ambiente.

De los resultados obtenidos, y con el objetivo de reducir el riesgo para la población y los ecosistemas, sería recomendable estudiar la disponibilidad y especiación de metales y metaloides en suelos y sedimentos para comprender mejor el ciclo de estos elementos y la transferencia de metales/metaloides de los suelos a la cadena alimentaria en la zona.

\section{Referencias}

Acosta J.A., MartínezMartínez S. Faz A. Millán R. Muñoz M.A. Terán T. Vera R. 2011. Characterization of the potential Mercury contamination in the apolobamba Gold Mining area, Bolivia. Spanish journal of soil science, 1, 86-99.

Alanoca, L., Maurice-Bourgoin, L. 2000. Estudio de la contaminación por mercurio en la cuenca alta del Río Tuchi. La Paz, Bolivia. La Paz, 234pp.

Anne, P. 1945. Sur le dosage rapide du carbone organique des sols. Annales de Agronomie, 2, 162-172.

Bowman, W.S., Faye, G.H., Sutarno, R., McKeague, J.A., Kodama, H., 1979. Soil Samples SO-1, SO2, SO-3 and SO-4-Certified Reference Materials. CANMET Report 79-3. CANMET Mining and Mineral Sciences Laboratories, Ottawa, ON.

Bower, C.A., Wilcox, L.V. 1965. Soluble salts. In: C.A. Black (ed.). Methods on Soil Analysis (933-940 pp). Madison, Wisconsin, U.S.A. American Society of Agronomy.

BWRHABTGG. 1995. Besluit van de Vlaamse Hegering Houdende Achtergrondwaarden. Bodernsaneringsnomen en Toepassingen van Gereinigde Grond. Bruselas. Belgium. Ministry of Environment and Employment, Brussels, 59 pp.

Campo, L. 2003. Componentes Programa Araucaria, resultados cartográficos. Programa Araucaria. 47 pp.

Chapman, H.D. 1965. Methods of Soil Analysis. Cation exchange capacity. In: C. A. Black (ed). (pp 891900). Madison, Wisconsin, U.S.A. American Society of Agronomy.

Choong, T.S., Chuah, T.G., Robiah, Y., Gregory, F.L., Azni, I. 2007. Arsenic toxicity, health hazards and removal techniques from water: an overview. Desalination, 217, 139-166.

Cobertera, E. 1993. Edafología aplicada: suelos, producción agraria, planificación territorial e impactos ambientales. Ed Cátedra. Madrid. Spain.

Duchaufour, P. 1970. Precis de Pedologie. Masson. Paris. $481 \mathrm{pp}$
Enríquez, J.C. 2001. Minería, Minerales y Desarrollo Sustentable en Bolivia. La Paz. Bolivia. Servicios Ambientales.

Ericksen, J.A., Gustin, M.S., Schorran, D.E., (...), Lindberg, S.E., Coleman, J.S. 2003. Accumulation of atmospheric mercury in forest foliage. Atmospheric Environment 37(12), 1613-1622.

FAO-ISRIC, 2006. Guidelines for Soil Description (revised), fourth ed. F.A.O, Roma. 97pp

Faz, A., Martínez, S., Acosta, J.A., Millán, R., Vera, R., Sierrra, M.J., García, G., Muñoz., M.A. 2003 Estudio sobre contaminación por actividades mineras en Apolobamba, Internal report. Cartagena, Universidad Politécnica de Cartagena-Agencia Española de Cooperación Internacional-Programa Araucaria. $260 \mathrm{pp}$.

Guan, X., Du, J., Meng, X., Sun, Y., Sun, B., Hu, Q. 2012. Application of titanium dioxide in arsenic removal from water: a review. Journal of Hazardous Materials, 215, 1-16.

Honorato, R. 2000. Manual de Edafología. Ediciones Universidad Católica de Chile. Santiago, Chile.

Horvat, M., Nolde, N., Fajon,V., Jereb, V., Logar, M., Lojen, S., Jacimovic, R., Falnoga, I., Faganeli, J., Drobne, D. 2003. Total mercury, methylmercury and selenium in mercury polluted areas in the province Guizhou, China. Science of the Total Environment, 304, 231-256.

Kabata-Pendias, A., Pendias, H. 2010. Trace Elements in soils and plants. Fourth edition CRC Press, Boca Raton.

Lechler, P.J., Miller, J.R., Lacerda, L.D., Vinson, D., Bonzongo, J.C., Lyons, W.B., Warwick, J.J. 2000. Elevated mercury concentrations in soils, sediments, water, and fish of the Madeira River basin, Brazilian Amazon: a function of natural enrichments? Science of the Total Environment, 260, 87-96.

Lindquist, O., Rhode, H. 1985. Atmospheric Mercury-a review. Tellus, 136-157

Litter, M.I., Morgada, M.E., Bundschuh, J. 2010. Possible treatments for arsenic removal in Latin American waters for human consumption. Environmental Pollution, 158(5),1105-1118.

Loayza, F., Franco, I. 2000. Estudio Ambiental, Socio Cultural y Económico de la Minería y la Comunidad en Bolivia, Perú y Chile. El Caso Boliviano. Centro Internacional de Investigaciones para el DesarroIlo, División Minería e Industria - Banco Mundial, La Paz. Bolivia.

M.D.S.M.A. (Ministerio de Desarrollo Sostenible y Medio Ambiente - Bolivia) 1996. Proyecto Piloto Oruro: Documento Plan de Gestión Ambiental. Swedish Geological AB, La Paz.

Malm, O., Pfeiffer, W.C., Souza, C.M.M., Reuther, R. 1990. Mercury pollution due to gold mining in the Madeira river basin. Brazil. AMBIO, 19 (1), 11-15.

Maurice-Bourgoin, L., Quiroga, I. 2001. Total mercury distribution and importance of the biomagnification process in rivers of the Bolivian Amazon. In: 
The Ecohidrology of South America Rivers and Wetlands (McClain M., Ed.), IAHS Special Publication $\mathrm{n}^{\circ} 6$, pp 49-66.

Maurice-Bourgoin, L., Quiroga, I., Guyot, J.L., Malm, O. 1999. Mercury pollution in the upper Beni River basin. Bolivia. AMBIO, 28 (4), 302-306.

Mulholland, D.S., Resende, G., Ferreira, D. 2012. Geological and anthropogenic influences on sediment metal composition in the upper Paracatu River Basin, Brazil. Environmental Earth Science 67, 13071317.

N.M.H.P.P.E. $1994 . \quad$ Netherlands Ministry of Housing. Physical Planning and Environment. Leidschendam. Holland.

Peech, M. 1965. Methods or Soil Analisis. Hidrogen-ion activity. (In C.A. Black (ed.). (pp 914-916) American Society of Agronomy. Madison, Wisconsin, U.S.A.

Peñaloza, R. y Reinhardt, J. 2000. Problemas ambientales de la minería Boliviana. Estudio de caso de la minería en el área de la ciudad de Potosí, Proyecto IDRC - WRI - CIPMA: Identificando los Impactos Económicos y Ambientales de la Liberalización del Comercio: Una Aplicación al Sector Minero, Fundación MEDMIN

Pfeiffer. W.C., Maim. O., Souza. C.M.M., Drude. L., Silveira. E.G., Bastos. W.R. 1991. Mercury in the Madeira River ecosystem, Rondonia, Brazil. Ecologycal Management, 38, 239-245.

Podwojewski, P., Poulenard, J., Nguyet, M.L., de Rouw, A., Nguyen, V.T., Pham, Q.H., Tran, D.T. 2011. Climate and vegetation determine soil organic matter status in an alpine inner-tropical saoil catena in the Fan Si Pan Mountain, Vietnam. Catena, 87, 226-239.

Risser, J.A., Baker, D.E. 1990. Testing soils for toxic metals. In: R. L. Westerman, pp 275-298 (Ed). Mad- ison, Soil Testing and plant analysis. Soil Sci. Soc. Amer. Spec. Publ. 3. 3rd Ed.

Soil Survey Division Staff. 1993. Soil Survey Manual. USDA Handbook 18, U.S.Government Printing Office, Washington, DC. $435 \mathrm{pp}$.

Soil Survey Staff, 2006. Keys to Soil Taxonomy, 10th ed. Natural resources Conservation service (NRCS), Washington DC.

Travnikov, O. 2005. Contribution of the intercontinental atmospheric transport to mercury pollution in the Northern Hemisphere. Atmos. Environ. 39:7541-7548.

USDA. 2003. National Soil Survey Handbook. Natural Resources Conservation service title 430-VI. Part 618.47. Reaction (pH): Soil Properties and Qualities.

W.R.B. 2006. World Reference Base for Soil Resources 2006. A framework for international classification, correlation and communication 103. Rome. 128 pp.

Wotruba, H., Hentschel, T., Livan, K., Hruschka F., Priester, M. 1998. Environmental Management in Small-scale Mining. Integrated Management of the environment in Small Mining (MEDMIN) e Swiss Agency for Development and Cooperation (COSUDE), La Paz.

Zang-Ho, S., Ki-Hyun, K., Min-Young, K., Meehye, L. 2005. Modelling study of reactive gaseous mercury in the urban air. Atmospheric Environment, 39, 749-761.

Zemmrich, A., Manthey, M., Zerbe, S., Oyunchimeg, D. 2010. Driving environmental factors and the role of grazing in grassland communities: A comparative study along an altitudinal gradient in Western Mongolia. Journal of Arid Environment, 74, 12711280.

Recibido: julio 2020

Revisado: diciembre 2020

Aceptado: enero 2021

Publicado: diciembre 2021 